\title{
Robotic lobectomy for non-small cell lung cancer (NSCLC): Long-term oncologic results
}

\author{
Bernard J. Park, MD, ${ }^{a}$ Franca Melfi, MD, ${ }^{\mathrm{b}}$ Alfredo Mussi, MD, ${ }^{\mathrm{b}}$ Patrick Maisonneuve, DipEng, ${ }^{\mathrm{c}}$ \\ Lorenzo Spaggiari, MD, ${ }^{\mathrm{d}}$ Ruy Kuenzer Caetano Da Silva, MD, ${ }^{\mathrm{e}}$ and Giulia Veronesi, $\mathrm{MD}^{\mathrm{e}}$
}

\begin{abstract}
Objective(s): We evaluated a large series of patients undergoing robotic lobectomy for the treatment of earlystage non-small cell lung cancer (NSCLC) to assess long-term oncologic efficacy.

Methods: A multi-institutional retrospective review of patients undergoing robotic lobectomy for NSCLC was performed. Robotic lobectomy was performed in a manner consistent with the Cancer and Leukemia Group B (CALGB) consensus video-assisted thoracic surgery (VATS) lobectomy technique using a robotic surgical system. Perioperative outcomes and long-term follow-up were recorded prospectively, and survival was calculated from the date of surgery to last follow-up.
\end{abstract}

Results: From November 2002 through May 2010, a total of 325 consecutive patients underwent robotic lobectomy for early-stage NSCLC at 3 institutions. The median age of patients was 66 years (range, 30-87 years), and $37 \%$ (120) were female. The majority were in clinical stage I (IA, 247; IB, 63). Conversion rate to thoracotomy was $8 \%(27 / 325)$. Overall morbidity rate was $25.2 \%$ (82/325), and major complication rate was $3.7 \%(12 / 325)$. There was 1 in-hospital death $(0.3 \%)$, and the median length of stay was 5 days (range, 2-28 days). Pathologic stage distribution was $54 \%$ (176) IA, $22 \%$ (72) IB, $13 \%$ (41) IIA, $5 \%$ (15) IIB, and 6\% (21) IIIA. With a median follow-up of 27 months, overall 5-year survival was $80 \%(95 \%$ confidence intervals $[\mathrm{CI}]=73-88)$, and by pathologic stage, $91 \%(\mathrm{CI}=83-99)$ for stage IA, $88 \%(\mathrm{CI}=77-98)$ for stage $\mathrm{IB}$, and $49 \%(\mathrm{CI}=24-74)$ for all patients with stage II disease. Overall 3-year survival for patients with stage IIIA disease was $43 \%(\mathrm{CI}=16-69)$.

Conclusions: Robotic lobectomy for early-stage NSCLC can be performed with low morbidity and mortality. Long-term stage-specific survival is acceptable and consistent with prior results for VATS and thoracotomy.

(J Thorac Cardiovasc Surg 2012;143:383-9)

Lung cancer remains one of the deadliest cancers worldwide. ${ }^{1}$ With aging and growth of the population combined with the persistence of cigarette smoking and the potential widespread adoption of computed tomographic lung cancer screening, ${ }^{2}$ there will be an increasing number of early-stage lung cancers appropriate for surgical resection. Lobectomy performed by minimally invasive video-assisted thoracic surgery (VATS) has proven to be feasible and oncologically acceptable for isolated non-small cell lung cancer (NSCLC). ${ }^{3-5}$ However, despite multiple studies showing this and clear benefits over thoracotomy, such as decreased length of stay, fewer postoperative complications, ${ }^{5-7}$

\footnotetext{
From the Division of Thoracic Surgery, ${ }^{\mathrm{a}}$ Hackensack University Medical Center, Hackensack, NJ; the Cardiac and Thoracic Department AOUP ${ }^{\mathrm{b}}$ University of Pisa, Pisa, Italy; the Division of Epidemiology and Biostatistics, ${ }^{\mathrm{c}}$ The European Institute of Oncology, Milan, Italy; the Division of Thoracic Surgery, ${ }^{\mathrm{d}}$ University of Milan, Milan, Italy; and the Division of Thoracic Surgery, ${ }^{\mathrm{e}}$ The European Institute of Oncology, Milan, Italy.

Disclosures: Authors have nothing to disclose with regard to commercial support.

Read at the 91st Annual Meeting of The American Association for Thoracic Surgery, Philadelphia, Pennsylvania, May 7-11, 2011.

Received for publication May 14, 2011; revisions received Oct 10, 2011; accepted for publication Oct 21, 2011; available ahead of print Nov 21, 2011.

Address for reprints: Bernard J. Park, MD, Division of Thoracic Surgery, Hackensack University Medical Center, 30 Prospect Ave, Suite 5636, Hackensack, NJ 07601 (E-mail: bpark@humed.com).

$0022-5223 / \$ 36.00$

Copyright (C) 2012 by The American Association for Thoracic Surgery

doi:10.1016/j.jtcvs.2011.10.055
}

decreased pain, ${ }^{8}$ and improved delivery and tolerance of adjuvant chemotherapy, ${ }^{9}$ VATS lobectomy has not become widely used. Indeed, a VATS approach has recently been reported to have been used in less than $6 \%$ to $20 \%$ of all lobectomies performed in the United States. ${ }^{10,11}$ The reasons for this are not entirely clear but may be related to technical limitations, such as 2-dimensional imaging and limited maneuverability of instrumentation.

A robotic surgical system has been developed to address some of these limitations. It has 3-dimensional, highdefinition imaging, instrumentation with 7 degrees of freedom, and a master-slave surgical cart (da Vinci; Intuitive Surgical, Sunnyvale, Calif). A handful of small series have reported similar techniques of robotic lobectomy showing acceptable perioperative results, ${ }^{12-15}$ but there is no large experience of totally robotic lobectomy evaluating both perioperative and long-term oncologic outcomes in patients with lung cancer.

The aims of this study are to evaluate a large cohort of patients who underwent robotic lobectomy to analyze both the perioperative and long-term survival results and determine whether it has an oncologically acceptable role in the surgical management of early NSCLC.

\section{METHODS}

A multicenter retrospective cohort trial was performed using prospectively collected data from the thoracic surgery divisions of 3 institutions: 


\section{Abbreviations and Acronyms \\ CALGB $=$ Cancer and Leukemia Group B \\ $\mathrm{CT}=$ computed tomography \\ NSCLC $=$ non-small cell lung cancer \\ VATS $=$ video-assisted thoracic surgery}

Memorial Sloan-Kettering Cancer Center, New York, New York, The European Institute of Oncology, Milan, Italy, and Ospedale Cisanello, Pisa, Italy. The study was approved by the institutional review board of each institution, and a data transfer agreement was made. Eligible patients were those with biopsy-proven or suspected primary NSCLC with no evidence of locally advanced or extrathoracic disease based on computed tomography (CT) of the chest, whole-body positron emission tomography/CT, and mediastinoscopy (select cases) who subsequently underwent attempted robotic lobectomy for primary NSCLC. Patients with carcinoid tumor, small cell lung cancer, benign or metastatic lesions, and those not undergoing lobectomy were excluded. Information regarding preoperative characteristics, operative details, hospital course, pathologic findings, and postoperative follow-up were recorded prospectively and sent to 1 institution (Milan) for analysis.

\section{Technique of Robotic Lobectomy}

At each institution the indications for robotic lobectomy are identical to those for VATS lobectomy-lesions isolated to the hemithorax, resectable by lobectomy, in patients with adequate cardiopulmonary reserve. Each surgeon (B.P., G.V., and F.M.) performed robotic lobectomy using a technique that conformed to the Cancer and Leukemia Group B (CALGB) 39802 consensus report for VATS lobectomy: use of non-rib-spreading incisions with the largest incision no greater than $8 \mathrm{~cm}$, videoscopic guidance, and traditional hilar dissection. ${ }^{16}$ The detailed technical aspects have been reported previously ${ }^{12,13,15}$ and will not be reviewed here. At 2 centers (Milan and Pisa) a 4-arm approach was used, whereas at the third center (New York) a 3-arm technique was employed. All patients underwent systematic mediastinal lymph node dissection. Patients gave written informed consent to undergo robotic surgery, and operative times were measured from first incision to closure. Conversion was defined as use of a rib-spreading thoracotomy at any point after docking of the robot to the patient and initiation of robotic dissection. Complications were recorded prospectively and categorized by the National Cancer Institute Common Terminology Criteria for Adverse Events version 3.0 (http:// ctep.cancer.gov/reporting/ctc.html) as either minor (grades 1 and 2) or major (grade 3 or higher). Clinical and pathologic staging was performed using the seventh edition of the TNM classification. ${ }^{17}$

\section{Surveillance and Follow-up}

At each institution patients were followed up postoperatively for lung cancer surveillance at least every 6 months for the initial 2 years with history, physical examination, blood work, and CT chest, and annually thereafter. Patients who did not return for follow-up evaluation were contacted by telephone, or their status was checked by the Social Security Death Index in the case of patients from the United States. Survival was calculated from the date of surgery to the date of death or last follow-up. Survival estimates were calculated with the Kaplan-Meier method and compared by the log-rank test. All analyses were performed with SAS statistical software version 8.2 (SAS Institute, Inc, Cary, NC).

\section{RESULTS}

From November 2002 through May 2010, 325 patients underwent robotic lobectomy for primary NSCLC at 3 centers. There were 123 consecutive patients from the center in New York during the study period, 82 patients from the center in Milan from November 2006 through May 2010, and 120 patients from the center in Pisa from January 2004 through May 2010. Table 1 shows the patient characteristics. There was a small preponderance of men, and $84 \%$ were former or current smokers. Over half of the procedures were upper lobectomies (right upper lobe, 92; left upper lobe, 75), and 1 patient underwent bilobectomy (right upper and middle lobes) for an upper lobe tumor invading across the horizontal fissure. The majority of cases were subtypes of adenocarcinoma ( $73 \%)$. Most patients had clinical stage I disease (IA, 247; IB, 63) and had no preoperative therapy. One patient had stage IIIA (N2) disease on the basis of mediastinoscopy and received induction chemotherapy. In all, 14\% (45/325) of patients underwent preresection mediastinoscopy.

Table 2 details the perioperative results of the robotic series. Median operative time was 3.5 hours. There were no intraoperative deaths, and the conversion rate to thoracotomy was $8 \%(27 / 325)$. The reasons for conversion are listed in Table 3. Three $(0.9 \%)$ patients had conversion for minor bleeding that did not require intraoperative or postoperative transfusion. Overall morbidity rate was $25.2 \%(82 / 325)$ and was not different between centers (New York, 26\%; Milan, $27 \%$; Pisa, $23 \%$ ). Twelve (3.7\%) patients had major complications, including bronchopleural fistula (2), pulmonary embolism (3), acute renal insufficiency (3), hemorrhage (2), and myocardial infarction (2). Supraventricular tachycardia was the most common postoperative complication, occurring in $37(11.4 \%)$ patients. Median chest tube duration was 3 days (range, 1-23 days), and length of stay was 5 days (range, 2-28 days). There was 1 in-hospital death in a patient in whom acute renal insufficiency developed followed by a pulmonary embolism and death on postoperative day 12 .

Seventy-six percent $(248 / 325)$ of patients had pathologic stage I disease (IA, 176; IB, 72), and in $68(21 \%)$ patients the severity of disease was upstaged (Table 2). The median tumor size was $2.2 \mathrm{~cm}$ (range, $0.7-10.2 \mathrm{~cm}$ ), and the median number of lymph node stations dissected was 5 (range, 2-8). Sixty-one (19\%) patients had metastatic nodal disease, and 67 patients received adjuvant cytotoxic chemotherapy. At a median follow-up of 27 months, $32(10 \%)$ patients had had recurrence, with 25 dead of their disease. The majority $(72 \%)$ of recurrences were distant (distant only, 17; locoregional+distant, 6), and 28\% (9/32) were locoregional only. Overall 5-year survival for the group was $80 \%$ (Figure 1, $A$ ), and stage-specific survival is shown in Figure 1, $B$.

\section{DISCUSSION}

With the continued growth and aging of the population and the likely acceptance of low-dose chest CT screening of high-risk populations, the number and proportion of 
TABLE 1. Patient characteristics

\begin{tabular}{lc}
\hline \multicolumn{1}{c}{ Category } & Result \\
\hline Age (y), median (range) & $66(30-87)$ \\
Male gender & $204(63 \%)$ \\
Smoking status & \\
$\quad$ Unknown & $3(1 \%)$ \\
Never & $50(15 \%)$ \\
Former/current & $272(84 \%)$ \\
FEV ${ }_{1}(\%$ pred), median (range) & $95(34-166)$ \\
DLCO (\% pred), median (range) & $87(11-196)$ \\
Primary tumor location & \\
RUL & $92(28 \%)$ \\
RML & $29(9 \%)$ \\
RLL & $71(22 \%)$ \\
RUL/ML & $1(0.3 \%)$ \\
LUL & $75(23 \%)$ \\
LLL & $57(18 \%)$ \\
Histology & \\
Adenocarcinoma & $239(73 \%)$ \\
Squamous cell carcinoma & $74(23 \%)$ \\
Other & $12(4 \%)$ \\
\hline$F E V_{l}$, Forced expiratory volume in the first second; $D L C O$, diffusing capacity of the \\
lung for carbon monoxide; $R U L$, right upper lobe; $R M L$, right middle lobe; $R L L$, right \\
lower lobe; $L U L$, left upper lobe; $L L L$, left lower lobe.
\end{tabular}

early-stage lung cancers diagnosed annually is likely to increase. Numerous reports from high-volume centers of excellence have shown that minimally invasive VATS lobectomy can be a feasible and safe alternative to thoracotomy lobectomy with additional benefits that include shorter length of chest tube duration and hospital stay, lower rate of major complications, decreased acute postoperative pain, and enhanced recovery and tolerance of adjuvant therapy. ${ }^{3-9}$ There are few, but increasing reports of robotic lobectomy using the purported benefits of 3-dimensional imaging and wristed instrumentation for minimally invasive

TABLE 2. Perioperative results

\begin{tabular}{lc}
\hline \multicolumn{1}{c}{ Category } & Result \\
\hline Operative time (min)* & $206(110-383)$ \\
Chest tube (d)* & $3(1-23)$ \\
Length of stay (d)* & $5(2-28)$ \\
Complications & \\
$\quad$ None & $243(75 \%)$ \\
Minor & $70(21.5 \%)$ \\
Major & $12(3.7 \%)$ \\
Perioperative mortality & $1(0.3 \%)$ \\
Pathologic stage $\dagger$ & \\
IA & $176(54 \%)$ \\
IB & $72(22 \%)$ \\
IIA & $41(13 \%)$ \\
IIB & $15(5 \%)$ \\
IIIA & $21(6 \%)$ \\
Tumor size (cm)* & $2.2(0.7-10.2)$ \\
Lymph node stations removed* & $5(2-8)$ \\
\hline *Median (range). †Seventh edition of the TNM classification.
\end{tabular}

*Median (range). $\nmid$ Seventh edition of the TNM classification.
TABLE 3. Reasons for conversion to thoracotomy

\begin{tabular}{lcc}
\hline & No. & Percent \\
\hline Incomplete fissures & 9 & 2.8 \\
Adhesions & 7 & 2.2 \\
Anatomic & 3 & 0.9 \\
Bleeding & 3 & 0.9 \\
Oncologic & 2 & 0.6 \\
Adenopathy & 1 & 0.3 \\
Tumor location & 1 & 0.3 \\
Loss of lung isolation & 1 & 0.3 \\
Total & 27 & 8.3 \\
\hline
\end{tabular}

lung resection. ${ }^{12-15}$ Previous robotic series have shown comparable perioperative results to those seen with VATS lobectomy, and our current experience again affirms that robotic lobectomy is feasible and safe with a short chest tube duration and length of stay, as well as low major morbidity $(3.7 \%)$ and in-hospital mortality $(0.3 \%)$ rates. The conversion rate to thoracotomy of $8.3 \%$ and the reasons for conversion were consistent with the largest series of VATS lobectomies, where the rate varies anywhere from $2.5 \%$ to $17.5 \%{ }^{3-5}$ Both conversion rates $(11 \%$ [17/ $150]$ vs $5.7 \%$ [10/175]) and median operative times (210 minutes vs 195 minutes) were lower after the first 50 cases at each center. This was quite similar to 1 author's (G.V.) previous experience demonstrating a 43-minute decrease in operative time after the initial 18 cases. ${ }^{15}$ No catastrophic episodes of hemorrhage were encountered, and bleeding episodes were easily managed through standard minimally invasive surgical strategies. In those cases of hemorrhage requiring conversion to thoracotomy, adequate control was obtained through the use of sponge stick tamponade through the utility incision while the robot was removed and the wound opened. In addition to feasibility data, our study has unique aspects not previously demonstrated.

First, it is the largest experience of totally robotic lobectomies reported to date. The first series reported by Melfi and coauthors ${ }^{12}$ contained 23 patients and focused primarily on technical aspects, as did a subsequent, larger series $(\mathrm{n}=34)$ from Park, Flores, and Rusch. ${ }^{13}$ Gharagozloo and colleagues ${ }^{14}$ described a series of 100 consecutive patients but used a hybrid VATS-robotic technique in which the mediastinal node and hilar dissection were performed robotically followed by conventional VATS ligation of the hilar structures.

Second, it is a multicenter, international experience with 1 center in the United States and 2 in Italy using similar patient selection criteria, surgical technique, and prospective evaluation of perioperative and long-term outcome. The patient population was relatively uniform, composed largely of patients with early-stage disease without prior treatment and with adequate cardiopulmonary reserve for lobectomy. 


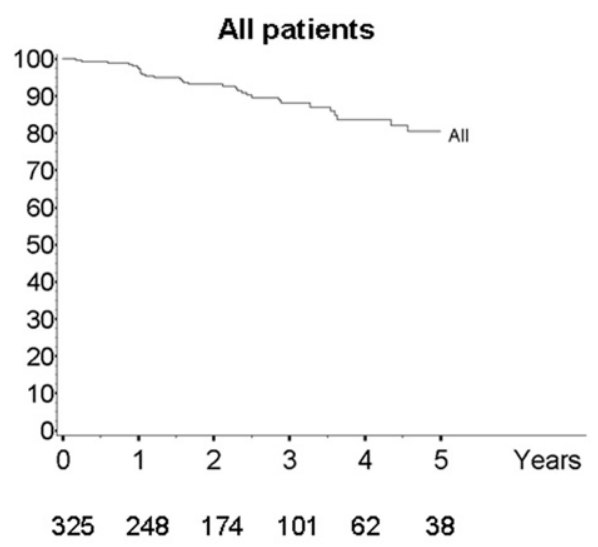

A
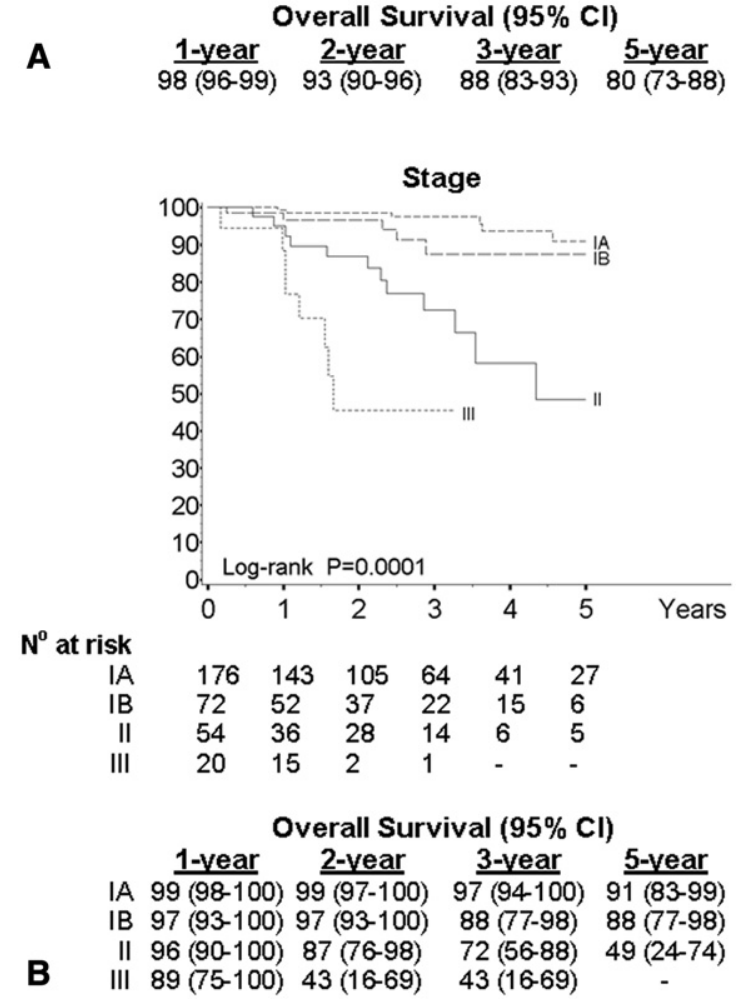

FIGURE 1. A, Robotic lobectomy overall survival. B, Stage-specific survival. $C I$, Confidence intervals.

As detailed in previous technical publications, each center used a fully robotic technique that conformed to the CALGB 39802 consensus criteria for minimally invasive lobectomy, ${ }^{12,13,15}$ and each of the authors performed systematic hilar and mediastinal lymph node dissection. In 2 of the centers a 4-arm technique was used (a fourth, small non-rib-spreading incision was used for placement of the fourth robotic arm for retraction), whereas a 3-arm approach was used at the third center. This minor difference in technique was likely of no clinical significance. Although the majority of tumors were small, there were larger lesions including a $10.2-\mathrm{cm}$ tumor. Because a non-rib-spreading utility incision was used, adequate retraction was able to be achieved easily either by robotic instrumentation alone or by the bedside assistant.

Third, this report is the first to look at the long-term oncologic outcome of robotic lobectomy for early NSCLC. The overall and stage-specific survivals are consistent both with the largest recent series of VATS lobectomies ${ }^{4,5}$ and the data used for the seventh edition of the lung cancer staging system, which was largely derived from conventional open surgery. ${ }^{17}$ Onaitis and colleagues ${ }^{4}$ reported 2-year overall survival of $80 \%$ and stage-specific survivals of $85 \%$ for stage I and $77 \%$ for stages II and higher in a cohort of 500 patients undergoing VATS lobectomy for clinical early-stage disease. Goldstraw and coauthors ${ }^{17}$ reported 5 -year overall survivals of $73 \%$ and $58 \%$ for stages IA and IB, respectively, and $46 \%$ and $36 \%$ for stages IIA and IIB. These data are very consistent with those observed in this large robotic series in which the 5-year survival for stage II patients was $49 \%$. The excellent 5 -year survival in pathologic stage I in this study (IA, 91\%; IB, $88 \%$ ) is likely related to the fact that the median tumor size was small $(2.2 \mathrm{~cm})$.

There are, however, limitations of this study and questions regarding the role of robotic technology in thoracic surgery. Inasmuch as this is a retrospective review, there are inevitable biases in patient selection and unknown differences between centers despite the fact that the patient characteristics and surgical techniques appear similar. Another limitation is the lack of other short- and long-term outcome measures, such as postoperative pain, respiratory function, rates of postthoracoscopy pain, and quality of life. Last, a comparative arm of VATS and/or thoracotomy patients is lacking. If use of robotic technology for thoracic surgical procedures increases, it will be important for future studies to attempt to discern differences between robotic and VATS approaches with respect to important outcomes, such as postoperative pain, quality of life, and cost.

With respect to the latter, cost of robotic technology in times of increasing health care expenditures is a real issue. There is no question that even without taking into consideration the amortized costs of these systems, robotics adds additional cost compared with conventional VATS. ${ }^{18}$ However, a recent analysis of the voluntary Society of Thoracic Surgery database demonstrated that although the percentage of all lobectomies done by VATS has been increasing, overall only $20 \%$ were performed by VATS during the 3 -year study period ending in $2006 .{ }^{10}$ Furthermore, there are some data, such as an even more recent analysis of a nonvoluntary national insurance database revealing that less than $6 \%$ of lobectomies were done by VATS, that suggest that the overall adoption rate of VATS lobectomy may in fact be lower in nonacademic, community-based settings. ${ }^{11}$ If robotic technology can lead to greater adoption of a minimally invasive approach in a safe and appropriate 
manner, the added cost may be justified by all the attendant benefits over traditional open surgery.

Robotic lobectomy is a feasible, safe, and oncologically sound surgical treatment for early-stage lung cancer. The technique is reproducible across multiple centers and yields results consistent with the best seen with conventional VATS. It should not be considered experimental, but an accepted minimally invasive thoracic surgical technique. Future evaluation of differences between robotic versus VATS versus thoracotomy approaches to thoracic diseases is warranted.

\section{References}

1. Jemal A, Bray F, Center MM, Ferlay J, Ward E, Forman D. Global cancer statistics. CA Cancer J Clin. 2011;61:69-90.

2. Aberle DR, Berg CD, Black WC, Church TR, Fagerstrom RM, et al., National Lung Screening Trial Research Team. The National Lung Screening Trial: overview and study design. Radiology. 2011;258:243-53

3. McKenna RJ Jr, Houck W, Fuller CB. Video-assisted thoracic surgery lobectomy: experience with 1,100 cases. Ann Thorac Surg. 2006;81:421-6.

4. Onaitis MW, Petersen RP, Balderson SS, Toloza E, Burfeind WR, Harpole DH, et al. Thoracoscopic lobectomy is a safe and versatile procedure. Ann Surg. 2006; 244:420-5.

5. Flores RM, Park BJ, Dycoco J, Aronova A, Hirth Y, Rizk NP, et al. Lobectomy by video-assisted thoracic surgery (VATS) versus thoracotomy for lung cancer. J Thorac Cardiovasc Surg. 2009;138:11-8.

6. Villamizar NR, Darrabie MD, Burfeind WR, Peterson RP, Onaitis MW, Toloza E, et al. Thoracoscopic lobectomy is associated with lower morbidity compared with thoracotomy. J Thorac Cardiovasc Surg. 2009;138: 419-25.

7. Cattaneo SM, Park BJ, Wilton AS, Seshan VE, Bains MS, Downey RJ, et al. Use of video-assisted thoracic surgery for lobectomy in the elderly results in fewer complications. Ann Thorac Surg. 2008;85:231-6.

8. Landreneau RJ, Hazelrigg SR, Mack MJ, Dowling RD, Burke D, Gavlick J, et al. Postoperative pain-related morbidity: video-assisted thoracic surgery versus thoracotomy. Ann Thorac Surg. 1993;56:1285-9.

9. Petersen RP, Pham D, Burfeind WR, Hanish SI, Toloza EM, Harpole DH, et al. Thoracoscopic lobectomy facilitates the delivery of chemotherapy after resection for lung cancer. Ann Thorac Surg. 2007;83:1245-50.

10. Boffa DJ, Allen MS, Grab JD, Gaissert HA, Harpole DH, Wright CD. Data from The Society of Thoracic Surgeons General Thoracic Surgery database: the surgical management of primary lung tumors. J Thorac Cardiovasc Surg. 2008;135: 247-54.

11. Gopaldas RR, Bakeen FG, Dao TK, Walsh GL, Swisher SG, Chu D. Video-assisted thoracoscopic versus open thoracotomy lobectomy in a cohort of 13,619 patients. Ann Thorac Surg. 2010;89:1563-70.

12. Melfi FMA, Ambrogi MC, Lucchi M, Mussi A. Video robotic lobectomy. MMCTS doi:10.1510/mmcts.2004.000448. Available at http://mmcts. ctsnetjounals.org/cgi/content/full/2005/0628/mmcts.2004.000448. Accessed May 1, 2011.

13. Park BJ, Flores RM, Rusch VW. Robotic assistance for video-assisted thoracic surgical lobectomy: technique and initial results. J Thorac Cardiovasc Surg. 2006;131:54-9.

14. Gharagozloo F, Margolis M, Tempesta B, Strother E, Najam F. Robot-assisted lobectomy for early-stage lung cancer: report of 100 consecutive cases. Ann Thorac Surg. 2009;88:380-4.

15. Veronesi G, Galetta D, Maisonneuve P, Melfi F, Schmid RA, Born A, et al. Fourarm robotic lobectomy for the treatment of early-stage lung cancer. J Thorac Cardiovasc Surg. 2010;140:19-25.

16. Swanson SJ, Herndon JE, D'Amico TA, Demmy TL, McKenna RJ, Green MR, et al. Video-assisted thoracic surgery lobectomy: report of CALGB 39802-a prospective, multi-institutional feasibility trial. J Clin Oncol. 2007;25:4993-7.

17. Goldstraw P, Crowley J, Chansky K, Giroux DJ, Groome PA, Rami-Porta R, et al. The IASLC Lung Cancer Staging Project: proposals for the revision of the TNM stage groupings in the forthcoming (seventh) edition of the TNM Classification of malignant tumours. J Thorac Oncol. 2007;2:706-14.
18. Park BJ, Flores RM. Cost comparison of robotic, video-assisted thoracic surgery and thoractomy approaches to pulmonary lobectomy. Thorac Surg Clin. 2008;18: 297-300.

\section{Discussion}

Dr Thomas A. D'Amico (Durham, $N C$ ). Robotics has been used in numerous surgical procedures, but, with few exceptions, this technologic advance has not translated into improved outcomes. Many robotic applications have been unveiled as the manifestation of a tool—sometimes a marketing tool—in search of an application. The principal advantages of robotic assistance in surgery are the greater degree of instrumental articulation and motion scaling. However, despite these recognized technical advantages, the demonstration of superior outcomes has been disappointing. As well, several disadvantages of the robotic system have been proven, including the lack of tactile feedback, personnel commitment, cost of the robot and its instruments, and the length of the procedures. In addition, the instrumentation that is currently available for use robotically is still limited.

The multi-institutional retrospective review by Park and his colleagues represents the largest robotic lobectomy series to date; more than 325 consecutive patients underwent robotic lobectomy for early-stage disease at the 3 institutions. The conversion rate was, admirably, relatively low, only $8 \%$, with reasonable morbidity, consistent with thoracoscopic lobectomy series in the literature. The length of stay was longer, perhaps with the inclusion of patients in Europe. Finally, stage-specific survival was excellent, also consistent with both open and thoracoscopic experiences, including patients with stage IIIA disease. Thus, the authors have demonstrated that robotic lobectomy for early-stage disease is effective, feasible, oncologically sound, and comparable, but not superior, to thoracoscopic lobectomy.

Less clear is the compared effectiveness of the robot vis-a-vis thoracoscopic lobectomy. Although thoracoscopic lobectomy has been demonstrated to be superior to open lobectomy in terms of outcomes and costs, analyses of the costs relating to robotic lobectomy suggest that the procedure is approximately $\$ 2000$ more expensive than open and $\$ 4000$ more expensive than VATS. In light of these issues, I have 3 questions, and I will ask them 1 at a time.

How should the cost of robotic lobectomy be considered in the evaluation of its effectiveness? Unlike some technologies in which cost has decreased over the evolution of the technology, the opposite seems to be happening with robotics; costs continue to increase, both fixed and variable. Although the promise that this technology as the future of all surgery has been adamantly put forward by others, I believe that a more logical response is required to adequately assess the effectiveness of robotic lobectomy.

Dr Park. Thank you for that analysis and question.

Cost is an extremely important feature, and you are right, robotics is a technology. I think it is critical to compare robotics (a minimally invasive modality) to thoracotomy, which is still the standard approach throughout the world. You stated that studies have shown that robotics is more expensive than thoracotomy, but I think that does remain to be seen and proven. We are going to be presenting a paper at the meeting of The International Society for Minimally Invasive Cardiothoracic Surgery (ISMICS) next month that compares costs associated with VATS versus robotic versus thoracotomy, which I believe will begin to address this. I 
think a critical issue is this: does robotics allowing us to transition from thoracotomy to minimally invasive approaches to early-stage lung cancer in the appropriate patients? Therefore, any cost analysis has to take that into account. If you are able to transition more surgeons into doing oncologically sound and safe procedures minimally invasively through the use of robotic technology, then perhaps those costs are justified. However, all things being considered, I think if you were to compare VATS nonrobotic and robotic in the most excellent hands, then yes, there's no question that robotics is more expensive. Whether that cost is justified depends on whether we could have most of our thoracic surgeons or people who do thoracic surgery do it by VATS.

Dr D'Amico. The issue of the transition brings up my second question. Inasmuch as thoracic surgeons, both in and out of training, are not adequately exposed to robotics, how do you recommend that surgeons take on this new technology to assure competency?

Dr Park. That question is in parallel to questions about advanced minimally invasive surgery in general. At most of our excellent training programs, the trainees are getting more and more exposed to advanced minimally invasive surgery, but that still leaves a whole population of surgeons who only know how to do thoracotomies, and they are faced with the same challenge of having to figure out how to do advanced VATS surgery, let alone robotic surgery. I think it is incumbent on major organizations, like The American Association for Thoracic Surgery and The Society of Thoracic Surgeons, to come up with guidelines for defining the appropriate use of such technologies and the minimum credentialing so that surgeons can get trained and be deemed to be qualified in these procedures. We need the organizations to come forth and define what the procedures are, to define how people can get trained in those procedures, and then to allow hospitals throughout the country and the world to determine how they can credential their surgeons and deem that they are qualified to do these procedures.

Dr D'Amico. I do not have an answer to my own question, but surgical associations have never done that for any other procedure, so I do not see The American Association for Thoracic Surgery or The Society of Thoracic Surgeons or the Board coming up and saying: This is how you need to do it. It is going to have to come from somewhere, but I would not necessarily expect that the associations would do it.

Finally, please comment on the impact of new robotic technology that is on the frontier: single-port entry, new energy sources, in-line staplers, infrared optics for sentinel node technology, simulation programs, and others. How do you think these new advances will translate into improved effectiveness for lobectomy?

Dr Park. I think simulation, as you mentioned, is one of the critical new technologies that is going to allow trainees or anybody who is established who wants to learn this technology and advanced minimally invasive techniques to do this in a more safe environment, to get practice without putting patients at harm. There is going to be stapling technology to allow direct control of the stapler. I think using the 3-dimensional optics is going to be a huge benefit, because even with some of the newer-technology staplers, most of us still feel some trepidation passing the staplers behind the hilar structures, and I think that is only going to help. Certainly, other instrumentation to help with retraction, exposure, and suctioning will all help. However, as you alluded to before, they are going to potentially result in increased costs. I think we have to evaluate these critically and decide which are necessary, and that will make these procedures safer and more feasible.

Dr Scott J. Swanson (Boston, Mass). What I would like to do now is just put on your hat of senior lung cancer surgeon. With the lung cancer screening trial coming and the fact that VATS lobectomies have gone from about $10 \%$ to $30 \%$, how would you use this technology across the country? Do you think it is realistic that all hospitals should have a robot and everyone should learn it? Should there be centers where people go for robotic surgery? How realistically should we get more minimally invasive operations to our patients?

Dr Park. We have to remember that the $30 \%$ number is from The Society of Thoracic Surgeons voluntary database. There are other nonvoluntary databases that show that some rates of VATS lobectomy are as low as $6 \%$. I am not sure that we are seeing an explosion of VATS lobectomy as a technique. I would love to think that that is true.

Your question is very similar to Dr D'Amico's.

As with any other technology, we need to identify centers of excellence and concentrate on trying to bring people to see what is going on in those centers, to organize training courses so that people can gain exposure to minimally invasive techniques and decide for themselves whether they are reasonable options and whether they do fit with their armamentarium. With increasing early-stage disease, increasing minimally invasive approaches is warranted and necessary, not only for the benefit of the hospital but for the benefit of the health care system in general in terms of allowing patients to recover quicker, getting them out of the hospital, and getting them back to their preoperative state.

Dr K. Adam Lee (Cherry Hill, NJ). In your data did you see any difference between a 3-arm approach and a 4-arm approach? Looking forward, do you see the robotic assisted technique transitioning an open thoracotomy surgeon to become more involved in minimally invasive surgery? Do you see some of those positive results of the robotics procedure helping to reach that goal?

Dr Park. There was no real difference between 3-arm and 4-arm techniques. Again, 2 of the centers had a 4-arm technique and 1 had a 3 -arm technique, but basically there was just 1 additional incision. All of the unified themes of non-rib-spreading and using videoscopic guidance were the same. There was no real difference clinically.

It is really hard to know whether robotics can help traditional thoracotomy surgeons transition to minimally invasive. I think we have to study that, if it is feasible, prospectively. I know, again, that VATS lobectomy and the excellent results that are published are being done so by essentially a core group of outstanding practitioners at great centers. The question is not whether robotics is the answer as opposed to VATS; the question is how we can appropriately use technology to increase the percentage of minimally invasive procedures that are done appropriately.

Dr Todd L. Demmy (Buffalo, NY). Another surgeon who performed many robotic lobectomies commented that a large tumor in the upper lobe is a hassle, and this gets to the issue with the robot having just 1 implementing arm per port. With thoracoscopy, you can use multiple retractors from a single port and handle that large tumor. In your study, did you notice any effect of big tumors in the upper lobe? You had a $10-\mathrm{cm}$ specimen. Was that in the lower lobe? 
Dr Park. All of the techniques had a utility incision and most of the assistants were able to retract through the utility incision. I am aware of no increased difficulty with larger tumors in these robotic series.

Dr Joachim Schirren (Wiesbaden, Germany). The patients in stage IA and 1B are nearly cured, but in stage II, you have results you can reach in open surgery in stage III. Therefore, my question is how many systemic relapses did you have and how many local relapses did you find in this stage?

Dr Park. Only 9 of the 32 patients had local-only relapse, and the majority of the 32 patients had either local plus distant or distant relapse. It was really systemic recurrence. 